\title{
LA INVESTIGACIÓN / INTERVENCIÓN PARA LA TRANSFORMACIÓN DE LA FAMILIA Y LA SALUD MENTAL*
}

Jairo G. Estupiñán Mojica**

\section{Introducción}

Esta separata muestra los avances de dos proyectos institucionales de investigación de la Maestría en Psicología Clínica y de Familia: "Vínculos, redes y ecología" y "Modelos de formación, terapia y consultoría en diversidad de contextos" hacen parte del Plan Institucional de la Investigación de Familla -PLANFA- (20002005); este plan está conformado por cuatro proyectos de investigación asumidos por equipos de profesores pagos por la Universidad, lo cual constituye un compromiso institucional de la Usta, de la Facultad de Psicología y de la Maestría en la sostenibilidad de la calidad académica del programa.
A continuación se presenta: un breve marco contextual del PLANFA y posteriormente algunos avances de los dos proyectos en mención.

\section{Contexto institucional de la investi- gación en la Maestría en Psicología Clínica y de Familia}

\subsection{En el orden institucional de la USTA}

En el orden institucional, el Plan de Investigación y sus proyectos correspondientes son coherentes con el PEI de la USTA, que propone "fortalecer los distintos programas de posgrado en marcha, mediante la consolidación y apoyo de una política de investigación diseñada con la impronta de la interdisciplinariedad y transdisciplinariedad, y eje-

\footnotetext{
Avances de dos proyectos institucionales de investigación en la Maestría en Psicología Clínica y de Familia en el marco del PLANFA

** Director de la Maestría en Psicología Clínica y de Familia, Universidad Santo Tomás.
} 
cutada bajo una acción eficiente de coordinación y cooperación intra e interfacultades. Esta interdisciplinariedad se dinamizará aún más mediante la realización conjunta de seminarios, encuentros, congresos y procesos investigativos de uno y otro género" (p. 90). Así mismo, sugiere "mantener inseparables los posgrados, la investigación, la proyección social y los planes de estudio de pregrado. Sobre todo en las maestrías y doctorados, los cuales deben tener como eje central la acción investigativa con sus respectivas líneas actualizadas y vinculadas al desarrollo local y regional. Es tarea prioritaria socializar sus planteamientos y resultados a la comunidad académica como mecanismo determinante para verificar su pertinencia y aceptación social. La evaluación de los planes y de sus productos investigativos han de ser una función permanente" (p. 91).

Desde esta perspectiva, la investigación en los posgrados se fundamenta en el paradigma ecosistémico de la familia, en el campo de la salud mental y en la búsqueda de los fundamentos de una psicología compleja/construccionista y constructivista, como alternativa de investigación/ intervención para mejorar la calidad de vida en términos de una convivencia saludable.

\subsection{Algunos referentes contextuales}

- La investigación en los Posgrados en Psicología y familia se fundamenta sobre el desarroIlo del PLANFA (Plan de Investigación Institucional en Familia). Este proyecto, diseñado para el período 2000 - 2005, fue aprobado por el Consejo de la Facultad y avalado por la Unidad de Investigación y de Pos-grados; reiteramos que en el momento actual el plan se desarrolla con base en cuatro proyectos institucionales, los avances de dos de ellos son el objeto de este texto.

- En su desarrollo histórico, los Posgrados en psicología y familia han venido generando un Modelo de Investigación / Intervención / In- vención, que articula las experiencias en la docencia, la investigación y el servicio social a la comunidad en consultorios de la USTA y en otros contextos institucionales con los cuales se han establecido convenios.

- El currículo de la Maestría en Psicología Clínica y de Familia desarrolla la investigación como un campo fundamental del proceso de entrenamiento de terapeutas e investigadores en el ámbito de la familia y la salud mental; los proyectos de tesis se desarrollan al interior de los cuatro proyectos; y los seminarios de investigación son escenarios/talleres para modelizar obras de conocimiento. Simultáneamente los equipos de estudiantes son asesorados por equipos de profesores investigadores, quienes son directores y codirectores de los proyectos institucionales y de los trabajos de grado; en este sentido, hay una clara diferenciación entre procesos investigativos institucionales y la llamada investigación académica. La política investigativa está plasmada en la aplicación del sistema de créditos académicos.

- A partir del 2000, se ha implementado un proceso de investigación/evaluación, asumido dentro del proyecto institucional de «Procesos de Formación» que ha generado una cultura de la evaluación, cuyos resultados nos han permitido ajustar los seminarios, los diseños generales de investigación, los protocolos de prácticas y los convenios interinstitucionales. Destacamos como tarea fundamental el estudio del impacto social y científico de nuestras prácticas en los consultorios de la Universidad.

- En lo referente a publicaciones, la última obra Construcciones en psicología compleja, publicada por la Editorial Universidad Santo Tomás, 2003, presenta los fundamentos paradigmáticos y epistemológicos desarrolla- 
dos por los posgrados en Psicología Clínica y de Familia.

- Los convenios interinstitucionales (ver su referencia específica en cada proyecto) se han actualizado en el 2003 y se han convertido en una fortaleza para la concreción de los proyectos de investigación y prácticas.

- Existe un equipo de docentes investigadores de tipo interdisciplinario de alta calidad profesional, cuyos currículos se presentan al final de este texto; ellos constituyen el capital social de este programa de trabajo.

\subsection{Denominación del campo/Línea de Investlgaclón}

Hemos definido nuestro campo/línea de investigación de la Maestría como: La investigación/ intervención para la transformación de la familia y la salud mental.

Mediante la creación de este campo del conocimiento se pretende la generación y co-construcción de distintos dominios sobre la familia, que enriquezcan su comprensión y evolución en las condiciones actuales de desarrollo del país y de formas de intervención novedosas para su transformación en función del bienestar de sí misma y de los miembros que la conforman.

El campo/línea de investigación se ha construido en coherencia con la perspectiva paradigmática general, al igual que con los intereses de la comunidad académica de los posgrados, respondiendo a los siguientes criterios:

- Definir los dominios de conocimiento y acción y proponer perspectivas de explicación y de comprensión de procesos específicos de la familia como sistema humano.

- Determinar campos específicos de investigación e intervención de la familia en contextos psicosociales particulares.
- Recrear la constelación paradigmática específica y los escenarios de la investigación, de tal manera que se expliciten las reglas de la actividad investigativa/interventiva particular.

- Definir objetivos y alcances del conjunto de proyectos y estudios que la conforman.

- Dar cuenta de manera permanente del estado del arte del campo de conocimiento y de la coordinación de los proyectos que la conforman.

\subsection{Objetivos generales del plan de Investigación en familla (PLANFA 2000 - 2005)}

- Comprender la complejidad del sistema familiar en su diversidad de contextos naturales y en su situación actual, con el fin de contribuir a la movilización de nuevas formas de organización y la conexión de éstas con los programas de intervención de las agencias sociales.

- Desarrollar abordajes conceptuales y metodológicos novedosos que se fundamenten en el conocimiento de las familias, en sus recursos, en sus capacidades auto-organizadoras, en sus pautas relacionales, en la diversidad de sus condiciones de composición en el contexto socio-cultural y la problemática particular.

- Describir las pautas que las familias mantienen en virtud de las distinciones que hacen del mundo y co-inventar los escenarios para viabilizar el cambio.

- Identificar y comprender las historias y narrativas dominantes de familias, instituciones y comunidades que le dan sentido a Ios síntomas y crear posibilidades alternativas de cambio. 
- Estudiar la "naturaleza" social de los vínculos y los modos de construcción de las redes sociales con que las familias interactúan e inventan las formas de convivencia humana.

- Crear diseños de investigación/intervención en donde emerja el cambio, considerándolo como metáfora de evolución, es decir, como un proceso co-construido, que no tiene relación directa con la resolución de problemas, sino con las historias que crean los problemas y cobran sentido en contextos específicos.

- Realizar publicaciones que den cuenta del trabajo investigativo del PLANFA.

\section{Proyectos específicos del PLANFA}

La investigación institucional en familia está constituida por los siguientes proyectos:

- Vínculos, redes y ecología.
- Historias familiares y narrativas en diversidad de contextos.

- Modelos de formación, terapia y consultoría en diversidad de contextos.

- Violencia familiar y ecología social.

\subsection{Enfoque integral y contextual de la formación de psicoterapeutas e in- vestigadores en el campo de la fa- milia y la salud mental}

Hemos consolidado, en la práctica, un enfoque integral y contextual de la formación y la investigación, que se presenta en el siguiente diagrama; este esquema opera de manera pragmática en todos los escenarios pedagógicos e interventivos. Lo hemos titulado círculos virtuosos del modelo de formación, en razón a su planteamiento sistémico, dejando ver con ello la dinámica interactiva y creativa de la acción humana de los procesos de formación.

Círculos virtuosos del modelo de formación

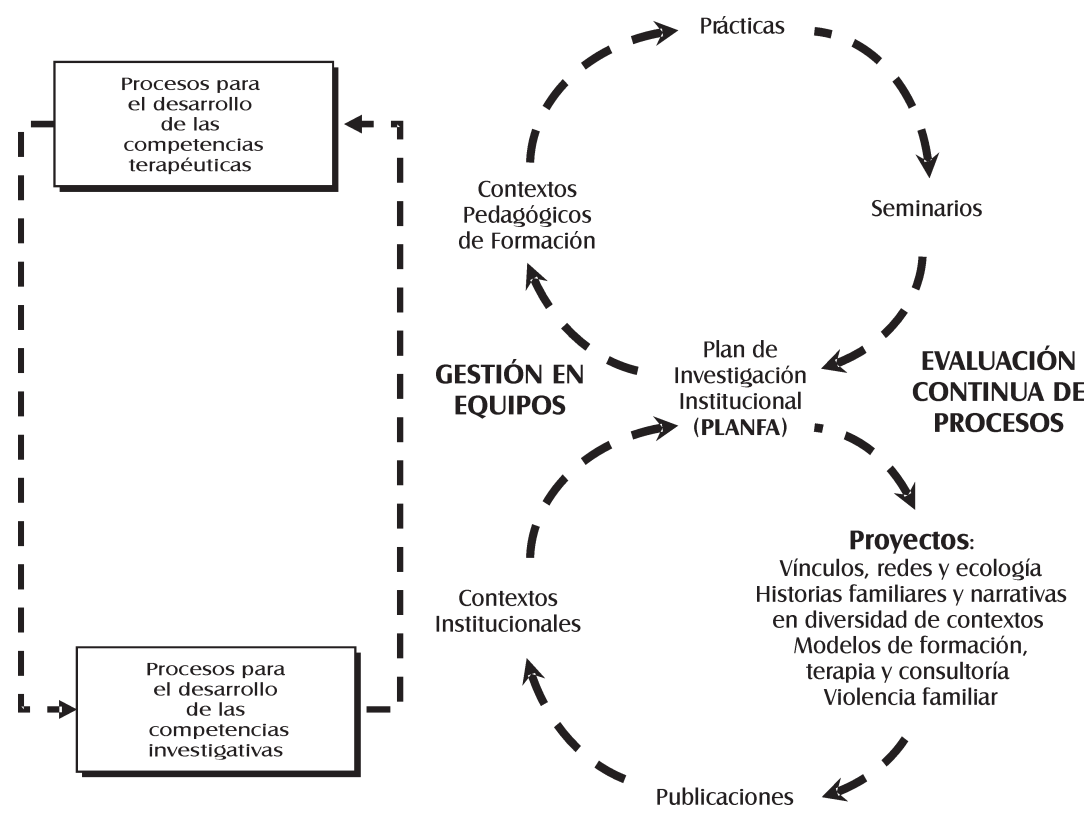

Como puede verse, el PLANFA se constituye en el nodo matriz de la información y de la gestión del currículo contextual de los posgrados. 
2.2 Sobre la articulación de los proyectos institucionales de investigación y de los trabajos de grado de los estudiantes

- El desarrollo del PLANFA, en su campo/línea y en los proyectos respectivos, es responsabilidad del equipo de investigación y se constituye en el contexto de desarrollo de los trabajos de grado de los estudiantes, de tal manera que actualmente todos los proyectos de grado de las últimas tres cohortes de egresados y de todos los estudiantes vigentes son cogestionados por los directores de los proyectos institucionales, quienes son sus directores.

- Los procesos de socialización de los trabajos de grado se han constituido en escenarios académicos que coadyuvan a su desarrollo y generan comunidad académica; de igual manera, las sustentaciones son muestras fehacientes de aplicación de diseños en el campo de la salud mental, los cuales son insumos claros para animar los estados del arte de los proyectos institucionales de investigación.

- Los cuatro proyectos inherentes al PLANFA se integran coherentemente en virtud de los siguientes criterios de formación:

- Desarrollar en los terapeutas y consultores la capacidad de autorreferencia en toda su expansión, desplegando así la utilización de sus recursos experienciales y, en general, de su historia familiar. Como proceso, la autorreferencia es básico para generar dominios de investigación e intervención.

- La capacidad para constituir equipos reflexivos es una competencia "clave" para la generación de contextos de intervención-investigación, pues la investigación implica, en el enfoque, intervención.
- El desarrollo del estilo personal como terapeuta o consultor e investigador, fundamentado en la estrategia de co-aprendizaje, viabiliza la internalización de las técnicas del enfoque sistémico con familias, en coherencia con los procesos de desarrollo humano de cada estudiante de los programas.

- La capacidad de meta-observación evoca y convoca un trabajo terapéutico e investigativo en redes sociales de forma que los síntomas se despliegan en el ecosistema.

- Los principios de rigor están asociados a los criterios de una epistemología de los sistemas observantes, que se basan en los procesos reflexivos, autorreferenciales, conversacionales y contextuales. La idea de fondo consiste en que la realidad es una construcción socio-cultural, de forma que compromete un diálogo riguroso con la tradición explicativa y comprensiva de los procesos psicológicos y el modo de abordarlos y de la apropiación concreta de aportes para las comunidades e instituciones humanas en términos de impacto.

- La investigación debe producir obras de conocimiento caracterizadas por aportar explicaciones de segundo orden; es decir, los datos y las explicaciones cualitativas deberán ser reflexionados por los actores de la investigación de modo tal que exista una toma de conciencia sobre dichas reflexiones que produzca explicaciones de tipo complejo.

- Los proyectos se articulan con los diversos escenarios de investigación y, por lo tanto, la investigación como práctica social desarrollará competencias básicas en la construcción del conocimiento en la perspectiva de segundo orden. En este sentido, dichos escenarios y procesos posibilitan el despliegue de las competencias comunicativas y reflexivas de 


\section{Universidad Santo Tomás}

los actores sociales del conocimiento y de los equipos de investigación conformados por estudiantes, profesores investigadores, familias y comunidades participantes.

- Se han definido indicadores de gestión y de impacto social y científico, los cuales se con- vierten, en el momento de ver la contribución de los proyectos, en elementos clave de retroalimentación del PLANFA. 